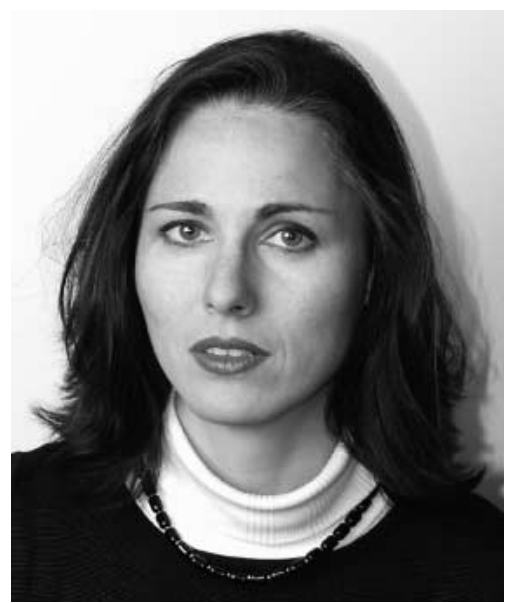

\title{
Chères lectrices, chers lecteurs
}

Vous avez en mains le premier numéro de l'année 2002, c.-à-d. de la première année faisant incontestablement partie du troisième millénaire, et, qui plus est, mon premier éditorial dans le Bulletin des médecins suisses. J'assume en effet depuis le ler janvier de cette année la charge de nouvelle secrétaire générale de la FMH. Licenciée en sciences politiques, mariée, j'ai 36 ans et un enfant.

Lorsqu'on m'a demandé si je voulais reprendre la succession du secrétaire général sortant, j'ai ressenti d'emblée une grande satisfaction sur le plan de mon cursus professionnel. Après avoir été élue officiellement par le Comité central, la tâche qui m'attendait m'a dans un premier temps fait quelque peu douter de moi. Mais en bonne économiste de formation, je me suis tout d'abord plongée dans la statistique de la maison: 28131 membres, 24 associations cantonales et 44 sociétés de discipline médicale, un Comité central à composition traditionnelle sous la houlette d'un président (et quel président!) et plusieurs dizaines de collaborateurs et collaboratrices dans divers départements. Ces chiffres ne disent pas grand-chose, en revanche, sur la nature de la FMH.

Je me suis donc attelée à la lecture de la littérature, des règlements et des rapports annuels, ainsi que des pages Internet du site officiel. J'ai pris connaissance de noms, de dates et d'événements et dî m'avouer que ma vision de la FMH ne s'éclaircissait pas pour autant. J'ai profité d'une autre possibilité d'en savoir plus en entrant dans les services de la FMH avant la remise officielle du flambeau, en tant que secrétaire générale "désignée", ce qui m'a permis de connaître l'organisation durant quatre mois "de l'intérieur". Je comptais bien mettre cette période à profit pour rassembler sur la FMH les connaissances indispensables à ma nouvelle fonction.

L'actualité n'a pas manqué d'être brûlante durant ces quatre mois d'initiation: levée de l'obligation de contracter, réorganisation de la formation médicale postgrade et continue, débats autour de la recherche sur les cellules-souche et de la solution des délais, législation sur les médicaments, sans oublier le TARMED jusqu'à plus soif! Cela ne m'empêchait pas de continuer à me demander ce qu'était véritablement la FMH. L'intérieur d'une structure, dans le bâtiment de l'Elfenstrasse? Se cachet-elle derrière une large échelle de valeurs, aboutissant, chez ses protagonistes, à un art protéiforme de la communication? Prend-elle ses assises sur sa longue expérience, accumulée au cours du centenaire de son existence? Est-elle la somme de ses membres? Ou leur produit?

Le premier janvier 2002 à minuit une, j'ai d̂u convenir ne pas encore pouvoir répondre à la question, ni au moment, probablement, où vous lirez ces lignes. Une seule chose me rassure: je ne suis pas la seule. Au cours de ma période de prise de contact avec la FMH, j'ai pris conscience du fait que les opinions et appréciations formulées à son égard étaient parfois diamétralement opposées. Quelle que soit la nature de la FMH aujourd'hui, il faudra qu'elle choisisse, parmi les nombreuses opinions, les plus prometteuses et les plus susceptibles de rallier la majorité et qu'elle les concrétise dans un climat qui ne peut que se durcir en permanence. Je pense que le défi à relever sera passionnant.

Je me réjouis de le vivre avec vous et vous souhaite un début d'année prometteur!

Annamaria Müller Imboden, Secrétaire générale de la FMH 